\title{
UNE ÉTRANGETÉ INVERSÉE: UN REGARD SUR LE THÉÂTRE CONTEMPORAIN
}

FrançoIse Dubor Universidade de Poitiers

\section{Résumé}

Alors que le théâtre contemporain se trouve accusé de dénaturation, par plusieurs types de spectateurs (critiques savants, amateurs occasionnels), l'examen de son évolution historique au XXe siècle révèle que les influences réciproques entre les arts lui ont notamment permis d'évoluer en transformant moins sa dépendance supposée au texte dramatique, en effet largement démontée, que ses modalités mêmes de représentation. Les attentes déçues des spectateurs font alors partie d'une dramaturgie où ceux-ci doivent accepter leur propre déplacement, leur mise en étrangement, en ostranenie, face à la représentation théâtrale conçue comme œuvre $\mathrm{d}^{1}$ art.

\section{Abstract}

Although contemporary drama now stands accused of distortion by several types of spectators (whether connoisseurs or occasional amateurs), the study of its twentiethcentury historical evolution has revealed that reciprocal influences among arts favoured its evolution, transforming not so much its supposed dependence upon the dramatic text - which has largely been shown - as its very modes of performance. Spectators' unfulfilled expectations are thus to be understood as part of some dramatic art in which they have to accept their decentring, their estrangement, their defamiliarization or ostranenie, when facing a dramatic performance conceived as a work of art.

\section{Mots-clés}

étrangement, représentation, texte, dramaturgie, distance.

\section{Keywords}

estrangement performance, text, dramatic art, distance. 
Si on veut parler d'étrangement au théâtre, on pense spontanément à l'effet $\mathrm{V}$, ou distanciation brechtienne, qui résulte d'une réflexion critique sur la pensée aristotélicienne de la représentation telle que nous en avons hérité des Classiques, c'est-à-dire sensiblement dénaturée. Si on considère que la vision d'Aristote de la tragédie se compose un siècle après la grande période des tragédies antiques, on peut élever au carré la dénaturation de l'objet du discours, de son approche intellectuelle, donc, qui signifie une distance à l'égard d'un objet propre à générer un étrangement de l'ordre de l'infidélité à l'objet en question. Comme les Classiques n'étaient pas en mesure d'évaluer l'écart entre Aristote et la tragédie antique, à laquelle ils ne pouvaient évidemment, pas plus qu'Aristote, avoir assisté, cette première distance ou infidélité, n'est pas apparue d'emblée de façon sensible. Et ils ont donc aggravé cette distance en interprétant Aristote qu'ils pensaient en réalité simplement relayer, dans leurs formulations théoriques. Notre vision de la tragédie procède de ce double malentendu, dont certains critiques ont récemment mis à jour le travail de distorsion - je pense à Florence Dupont ${ }^{1}$ - qui met à jour un héritage singulier car il doit finalement peu à l'original.

On peut se demander sur quel Aristote se fonde Brecht pour élaborer sa critique. Comme il travaille sur la conception aristotélicienne de la représentation, plutôt que de la tragédie, qui est un terrain d'application singulier, on a tendance à penser qu'il est revenu à la source même, mettant donc de côté les lectures, donc les interprétations dont nous avons héritées. Les principaux textes de Brecht où il expose sa théorie anti-aristotélicienne, désignée comme "théâtre épique", sont L'Achat du cuivre (1937) et le Petit Organon pour le théâtre (1956). Il s'agit d'enrayer le phénomène trop hâtif de la reconnaissance pour faire place à un questionnement critique, chez le spectateur dont il s'agit donc de dé-placer le regard, en élaborant une théorie de la représentation où la distanciation agit comme un principe esthétique opératoire sur une réception critique: si cette distanciation est opératoire, c'est bien parce qu'elle travaille à rendre la reconnaissance problématique, parce qu'en fin de compte, il s'agit pour Brecht d'interrompre le processus d'approbation immédiate du spectateur - de repenser les fondations de la rhétorique dramatique. Ainsi, pour lui, cet acte esthétique a une portée politique: donner une perception étrange d'un objet familier vise à désaliéner le spectateur en lui fournissant un nouveau positionnement, en exerçant sa conscience critique. Et de ce fait, l'œuvre d'art elle-même passe du terrain du procédé esthétique à celui de la responsabilité idéologique. A cette fin, Brecht conçoit une dramaturgie comme une machine de guerre, qui certes s'applique à toutes les données constitutives du théâtre (la fable, le décor, la gestualité, la diction, bref, le jeu de l'acteur, jusque dans ses adresses directes au public) mais il s'attaque de ce fait, prioritairement, à une théorie de la représentation. Tandis que le grief principal de Florence Dupont envers Aristote touche au fait qu'il ait imposé la priorité du texte, au théâtre, au mépris de toutes les données proprement scéniques, donc théâtrales, à cet art devenu à cause de lui principalement littéraire.

Même si le terme brechtien de distanciation provient directement de l'ostranenié, terme de Chklovsky qui nous réunit aujourd'hui, je voudrais plutôt vous parler de théâtre contemporain, en croisant les deux préoccupations de Brecht et de Florence Dupont, concernant ces deux notions que sont le texte et la représentation.

Le texte a en effet fondé une tradition théâtrale dont nous avons hérité en tant que spectateurs, c'est-à-dire en tant que destinataires de l'œuvre d'art théâtrale. Et la représentation évolue au rythme de l'évolution de ses codes qui sont aussi les nôtres, mais qui, précisément, s'acharnent à nous dé-placer à mesure qu'ils se transforment. C'est de notre relation à la représentation qu'il est aussi question dans mon propos. Et pour parler de théâtre, il faudra bien aussi d'autres arts: non seulement ceux qui sont

${ }^{1}$ DUPONT, Florence. Aristote ou le vampire du théâtre occidental. Paris, Aubier, 2007. 
convoqués sur la scène théâtrale, mais aussi ceux qui ont influé sur la conception de la représentation théâtrale, même s'ils ne sont pas formellement présents sur scène.

Le théâtre contemporain a fait l'objet d'une analyse critique allemande, qui se concentre sous la formulation de théâtre post-dramatique. ${ }^{2}$ Passons sur l'usage et/ou le sens problématique de l'adjectif, ${ }^{3}$ pour nous concentrer sur la thèse de Lehmann, censée définir un «nouveau» théâtre, à propos de théâtre contemporain. Le constat global concerne le primat de l'image sur le texte, sans que nous soyons renvoyés au théâtre spectaculaire à vertu divertissante qui traverse le XIXe siècle. ${ }^{4}$ Le propos de Florence Dupont à propos d'Aristote s'inscrit dans cette ligne qui examine de manière critique la façon dont le texte s'est imposé dans le théâtre occidental. Lehmann évoque un théâtre où s'opère la transgression des genres: cette dernière se circonscrit dans la traversée du "genre" théâtre par les "genres" suivants, la chorégraphie, les arts plastiques, le cinéma, la musique. L'ouvrage date de 1999 en Allemagne, traduit en 2002 en français, et a produit une réaction de défiance critique quelquefois violente, souvent polémique, où certains ont cru bon de venir défendre la pérennité du théâtre de texte (Jean-Pierre Sarrazac défendant son Avenir $d u$ drame, produit vingt ans auparavant, en 1981, lui-même adossé à l'ouvrage de Peter Szondi, La Théorie du drame moderne, 1956, à propos de la période 1880-1950). Or si tous deux traitent en effet du théâtre vu depuis le texte, globalisé sous le mot vague de drame, le texte sur la scène théâtrale, et depuis la poésie notamment, a grandement changé de figure. Pour preuve, cet extrait de Pierre Reverdy, un poème en prose de 1915:

\section{Traits et figures}

Une éclaircie, avec du bleu dans le ciel; dans la forêt, des clairières toutes verbes; mais dans la ville, où le dessin nous emprisonne, l'arc-de-cercle du porche, les carrés des fenêtres, les losanges des toits.

Des lignes, rien que des lignes, pour la commodité des bâtisses humaines.

Dans ma têtes des lignes, rien que des lignes - si je pouvais y mettre un peu d'ordre seulement.

Il ne suffit donc pas de renvoyer aux calligrammes d'Apollinaire ou à la typographie mallarméenne pour montrer comment le texte gagne une dimension plastique - esthétique. Reverdy en fait la forme de sa propre poétique et la thématise, sous l'effet du cubisme de son époque. Il y a des chances pour que le théâtre présente une mixité intrinsèque comparable. Je ne prends pas cet exemple poétique pour souscrire à mon tour au diktat du texte, mais pour montrer combien la réception a pris acte, en littérature, de ce type de transformation sans que sa défiguration supposée ou le risque de sa perte ne soit brandi avec véhémence: il ne me semble pas que quiconque ait jamais perçu le risque de voir la poésie se perdre ou se fondre dans des innovations jugées dénaturantes. La littérature (poétique) est ici un simple moyen de s'extraire des effets de comparaisons habituels du théâtre contemporain avec d'autres arts de la scène ou arts plastiques. Je dis que le phénomène d'étrangement au théâtre, perçu tel aujourd'hui, est pourtant présent dans tous les arts, ni plus ni moins, et en littérature ou même en poésie aussi.Il faudrait plutôt, sans doute, se demander pourquoi cela pose un problème particulier dans la réception du théâtre. Pourtant, un tel terrain, préparé depuis plus d'un siècle, déroute par exemple le public du festival d'Avignon, qui exprime en 2005 un désaveu sonore et continu, cherchant en vain dans ce théâtre perdu à force d'être dénaturé: le texte, où est le texte? - comme

\footnotetext{
${ }^{2}$ LEHMANN, Hans-Thies. Le théâtre postdramatique. Paris: L'Arche, 2002.

${ }^{3}$ Voir BIDENT, Christophe. "Et le théâtre devint post-dramatique: histoire d'une illusion", Théâtre / Public, $\mathrm{n}^{\circ} 194$, 2009, p. 76-82.

${ }^{4}$ Voir EL NOUTY, Hassan. Théâtre et pré-cinéma: essai sur la problématique du spectacle au XIXe siècle. Hassan el Nouty. Paris: A.-G. Nizet, 1978.
} 
Mortin, dans L'Hypothèse de Pinget cherchait l'auteur - où est l'auteur? Il en est ainsi, cependant à chaque défiguration du texte, qui en a enregistré un certain nombre: Ionesco, Beckett, Sarraute, Duras, sont mal digérés. On acclame Novarina ou on s'en détourne - il est rarement compris par le spectateur moyen (l'amateur occasionnel, non spécialiste). Si on y regarde un tant soit peu, donc, l'espace du texte est défiguré non moins que la peinture qui s'est éloignée de la figuration en entrant dans l'abstraction. C'est que, précisément, tous les enjeux de l'étrangeté se concentrent en définitive sur la notion de représentation.

Lehmann postule quelques éléments définitionnels pour son théâtre postdramatique, qui sont en effet des mignes majeures de la représentation théâtrale contemporaine. Le "théâtre dramatique" qui le précède chronologiquement se fonde, dit-il, sur des catégories que le postdramatique a abandonnées: l'imitation, l'action, la production d'un lien social qui génère un effet cathartique, en donnant le primat au texte. C'en serait fini du théâtre créateur d'illusion.

Selon lui, une césure s'est produite dans les années 1970: les avant-gardes n’ont pas empêché la prospérité du théâtre de texte, et n'auraient donc pour cette raison pas part à cette césure; elles auraient agi sur des éléments plutôt que sur l'ensemble de la représentation théâtrale, et elles seraient restées fidèles à une mimesis et à une action sur la scène. Ce serait plutôt l'envahissement de la vie quotidienne par les médias qui auraient promu le surgissement dans ces années 1970 d'une nouvelle pratique du discours théâtral, sous des formes diverses. Mais il admet pour finir l'existence d'anticipations du théâtre postdramatique au début du XXe siècle. Cela fait beaucoup de fonctions et d'opérations, progressivement contradictoires, en ce qui concerne les avant-gardes théâtrales du début du XXe siècle. L'agencement de paradigmes en constellation (p. 32) serait décisif pour l'entrée dans le postdramatique, qui se présente comme point de rencontre des arts (p. 41):

de ce fait, [il] développe un potentiel de perception qui se détache du paradigme dramatique (et qui plus est, de la littérature). Il n'est pas surprenant qu'avec cette forme de théâtre, les adeptes d'autres disciplines (arts plastiques, danse, musique) soient souvent plus à l'aise que les spectateurs inconditionnels du théâtre narratif littéraire. (p. 41)

Ce nouveau théâtre opèrerait une tabula rasa de deux grandes notions: la mimesis et la fable. La fin de la mimesis serait, après Artaud, la volonté d'écarter du théâtre la redondance, le double du réel, pour présenter au lieu de représenter. La fable, pour exister, n'a pas nécessairement besoin de narration explicite et linéaire... Aussi existe-t-elle toujours. Il faudrait dire: aussi co-existe-t-elle toujours. La disparition de ce triangle: drame - action - imitation (p. 50) avait bien été théorisée par Artaud dans les années 1930 (p. 42)...

Les prises de position de Lehmann, valeureuses, en ce qu'il tente d'aborder frontalement - enfin! - un état des lieux de la création théâtrale contemporaine, s'annulent hélas quand au fil des pages il en vient à dire une chose et son contraire, déniant la parenté du théâtre contemporain avec les avant-gardes du début du XXe siècle, tout en affirmant la dette de celui-là envers celles-ci, postulant l'autonomie du théâtre contemporain, mais ayant recours à Artaud pour en expliquer les grands enjeux... Il manque des lignes de filiation dans le travail des critiques - a fortiori chez les spectateurs. A peine voit-on Madeleine Mervant-Roux (r)établir le lien de Claude Régy au théâtre symboliste - avec raison.

En vérité, les échanges de paradigmes auxquels les artistes se sont livrés pour penser leur art se sont multipliés depuis le début du XXe siècle, et depuis ses avant-gardes. Le peintre Kandinsky parle de peinture avec des termes - des outils - de musique (ton, hauteur, intensité, rythme). On a vu Reverdy déployer sa poétique en termes de peintre. En réalité, la distinction de Lessing entre les arts du temps et les arts de l'espace s'est brouillée dans cette époque inaugurale, et il faudrait dire ce que les arts plastiques 
doivent au théâtre justement, ${ }^{5}$ en particulier en termes d'introduction de la temporalité dans les œuvres, dont procèdent non seulement la performance et le happening, mais aussi la sculpture, livrée à l'éphémère dans le land art, par exemple. ${ }^{6}$ Le mouvement est aussi un emprunt au théâtre, d'où les mobiles, toujours à propos de sculpture, ou les bien nommés "théâtres d'artistes", qui concernent notamment le même Calder, entre autres artistes.

Toute compréhension littérale du sens est un symptôme de pauvreté intellectuelle, de dénuement en matière d'outils théoriques. Il est certes manifeste que la "rencontre des arts" sur scène caractérise une bonne part de la création contemporaine. Pour autant, la question de leur hiérarchie n'est pas plus pertinente que celle qui, en littérature, ordonnerait la singularité de la poétique de l'auteur et son univers intertextuel - ou ses références à d'autres arts. Ce n'est pas parce qu'on trouve des écrans sur scène qu'il s'agit d'organiser la part du théâtre et celle de la vidéo - ou du cinéma, ou de la photographie, selon les cas. Les procédés cinématographiques du collage, du montage, du cadrage, sont apparus sur la scène théâtrale dans les années 1920, sous l'influence conjointe et un peu tardive du cubisme (la peinture cubiste) - dont on retrouve la trace prégnante dans des films de cinéma expérimental des mêmes années, réalisés par des peintres... Ainsi le Ballet mécanique de Fernand Léger (1924). Tous les nouveaux outils - notions et concepts comme technologies diverses - nécessitent un temps d'expérimentation et d'apprentissage, et l'écran sur la scène théâtrale, pour reprendre cet exemple, a d'abord parasité la seule présence de l'acteur.

Le problème de la réception du spectateur s'est manifesté sensiblement quand celui-ci a cru perdre la forme conventionnelle à laquelle il s'attendait: le cinéma sur la scène théâtrale, le théâtre sur la scène chorégraphique, l'installation plastique à la place du théâtre... Les arts co-habitent, dans un dialogue ininterrompu qui appelle leur présence patente ou reste en-deçà des œuvres, les précédant et créant de ce fait un précédent. De fait, ils adviennent en un lieu où on ne les attendait pas nécessairement.

C'est là, sans doute, que prend place le sentiment d'inquiétante étrangeté - inversé: le spectateur n'est pas face à un objet familier qui progressivement sort de ses gonds; tout au contraire, cet objet déroute d'emblée et oblige le spectateur à parcourir un chemin auquel il ne s'attendait pas, par lequel il pourra réapproprier cet objet à ses attentes préalables. Les changements enregistrés dans les codes de représentation ont pris une certaine amplitude: il ne s'agit plus seulement de considérer la façon dont un acteur semble, par sa parole monologale, harponner le public en s'adressant frontalement à lui, comme dans certains spectacles de TG STAN, alors que la séparation scène / salle existe toujours, et reste efficiente. Dans ce cas, le classique interdit de l'adresse directe s'effrite largement, mais la convention qui distingue acteur et spectateur garde toute sa validité - elle est remise en cause dans d'autres types de représentation théâtrale, avec le Living Theatre, notamment.

Il faut plutôt considérer une chorégraphie dans laquelle les danseurs semblent avoir renoncé à danser - mais ils se meuvent dans l'espace scénique, étrangers à toute virtuosité, égrénant un texte qui passe d'un danseur à l'autre (c'est Description d'un combat, Maguy Marin, 2009): les codes de la chorégraphie empruntent ceux du théâtre, l'attente du spectateur, en termes de danse, est donc déçue. Ou encore: un spectacle de théâtre nous donne à voir un travail de bruitage généralisé du plateau (c'est l'adaptation et la mise en scène du roman de Sebald, Les Anneaux de Saturne, par Katie Mitchell en 2012); ou encore un concert exceptionnel de pièces rares composées par des musiciens ayant subi les camps nazis, et n'y

\footnotetext{
${ }^{5}$ Cf. FALGUIERES, Patricia. “Aire de jeu. À propos du théâtre et des arts au XXe siècle”. In Cahiers du Musée national d'Art Moderne, 101 (automne 2007), p. 62-85.

${ }^{6}$ L'argument est avancé par ROSENBERG, Harold. The De-Definition of Art. N. York: Horizon, 1972.
} 
ayant pas nécessairement survécu, en guise de théâtre (c'est Derniers jours. Une veillée de Christoph Marthaler, 2013).

Le fil rouge de ces spectacles contemporains, de ces représentations en apparences éclatées, écartelées, dénaturées, c'est la dramaturgie, c'est le travail de construction du sens, au prix des moyens fournis qui déçoivent l'attente de moyens convenus. Ce n'est pas un hasard si Brecht a tant insisté sur cette étape du travail qui convoquait hautement toutes les énergies de son équipe.

Contrairement à ce que certains critiques ont avancé, il ne s'agit pas de hiérarchiser ni de déhiérarchiser les arts sur la scène. Il s'agirait plutôt, peut-être, de l'effet unificateur de la scène sur l'art ou sur les arts qui la requièrent - arts de la scène ou non, par conséquent - dans cette convocation qui se donne l'objectif d'une représentation qui réinvente sa grammaire selon les nécessités de sa dramaturgie propre. Il ne s'agit pas non plus d'expérimentation: elle est largement explorée depuis le début du XXe siècle. Il s'agit plutôt d'abandonner un certain nombre de frontières au bénéfice de quoi le théâtre a pu devenir un art de l'espace plus que du temps. A moins que toute œuvre, scénique ou non, s'engage dans la voie de l'éphémère, du présent singulier de sa propre performance. Mais aujourd'hui, de cet éphémère, nous gardons la trace, et cette trace même fait œuvre, permettant à l'original de durer, d'entrer dans l'intemporel. Le théâtre contemporain participe de ce mouvement général, de plus en plus. Simplement, la trace n'est plus le texte, nécessairement, mais vidéo, ou livre - mais c'est alors un livre qui accompagne les errements de la création (comme pour Seuls de Wajdi Mouawad, 2008 - le principe est identique pour la conception qu'a Sophie Calle du livre). L'écriture de la représentation n'est plus celle du texte du drame.

Les autres arts ont investi l'espace à trois dimensions et le présent immédiat du jeu qui caractérisaient le théâtre. Les spectateurs en sont quelquefois distancés. Il me semble donc que le théâtre a, sinon imposé, du moins fourni aux autres arts, ses propres modalités de représentation, ou la matière permettant à ces autres arts de repenser les modalités de leur propre représentation. Au début du XXe siècle, la musique a pu jouer un tel rôle. Dans un tel processus, le théâtre lui-même s'en trouve transformé - mais ni dénaturé, ni méconnaissable: art de la présence et de l'espace, le suspens qu'il impose au spectateur serait donc aujourd'hui moins de sa fable que de sa forme: il impose donc au spectateur le travail inattendu de sa redéfinition sisyphéenne, à proportion de la perpétuelle réinvention de sa propre dramaturgie. Le spectateur, ainsi, n'est plus dans son fauteuil, mais sur la route, en itinérance. L'ostranenie est comme son nouveau territoire. 